\title{
Correction: Melatonin-induced ApoE expression in mouse astrocytes protects endothelial cells from OGD-R induced injuries
}

Jun Xiang (D, Wen Zhu, Feng Yang, Zhong-Hai Yu, Min Cai, Xiang-Ting Li, Jing-Si Zhang, Wen Zhang and Ding-Fang Cai (1)

Correction to: Translational Psychiatry https://doi.org/10.1038/s41398-020-00864-9 published online 8 June 2020

This Article was updated shortly after publication in order to amend co-author Jing-Si Zhang's affiliations (Dr Zhang was erroneously associated with affiliations 1 to 4 rather than just affiliation 1 as is correct) and various typographical errors in superscripts and subscripts.

Published online: 17 July 2020 